\title{
Thioredoxin and thioredoxin reductase: Current research with special reference to human disease
}

\author{
Arne Holmgren *, Jun Lu \\ Division of Biochemistry, Department of Medical Biochemistry and Biophysics, Karolinska Institutet, SE 17177 Stockholm, Sweden
}

\section{A R T I C L E I N F O}

\section{Article history:}

Received 10 March 2010

\section{Keywords:}

Thioredoxin

Thioredoxin reductase

Redox regulation

Thiol

Disulfide

Selenocysteine

\begin{abstract}
A B S T R A C T
Thioredoxin (Trx) and thioredoxin reductase (TrxR) plus NADPH, comprising the thioredoxin system, has a large number of functions in DNA synthesis, defense against oxidative stress and apoptosis or redox signaling with reference to many diseases. All three isoenzymes of mammalian TrxR contain an essential selenocysteine residue, which is the target of several drugs in cancer treatment or mercury intoxication. The cytosolic Trx1 acting as the cells' protein disulfide reductase is itself reversibly redox regulated via three structural Cys residues. The evolution of mammalian Trx system compared to its prokaryotic counterparts may be an adaptation to the use of hydrogen peroxide and nitric oxide in redox regulation and signal transduction.
\end{abstract}

(ㄷ) 2010 Elsevier Inc. All rights reserved.

\section{Introduction}

Thioredoxin (Trx), together with thioredoxin reductase (TrxR) and NADPH comprising the thioredoxin system, was discovered by Peter Reichard and coworkers in 1964 as a hydrogen donor for enzymatic synthesis of cytidine deoxyribonucleoside diphosphate by ribonucleotide reductase from Escherichia coli [1]. Enzyme purification also resulted in a highly pure TrxR as a flavoprotein with specificity for reduction of the active site disulfide in oxidized Trx [2]. The amino acid sequence of $E$. coli Trx 1 with 108 residues was determined in 1968 [3] demonstrating the universally conserved active site -Cys-Gly-Pro-Cys-. The three-dimensional structure of oxidized Trx, crystallized as a cupric ion complex, was discovered in 1975 [4] establishing the Trx fold, today a major protein structural element. By studies of the mechanism and kinetics of the Trx, it became clear that it is the cells' major protein disulfide reductase potentially being the physiological equivalent of a reducing agent like dithiothreitol [5]. Characterization of an E. coli mutant lacking Trx1 [5] resulted in the discovery of glutaredoxin as a GSH-dependent hydrogen donor for ribonucleotide reductase with overlapping functions to Trx in many systems [5,6].

Research on Trx and TrxR through efforts in many laboratories worldwide today covers large areas of biomedicine. There are more than 6100 references in PubMed and e.g. plant biochemistry demonstrates that there are more than 20 genes encoding isoforms of Trx regulating photosynthesis and other plant biochemical path-

\footnotetext{
* Corresponding author.

E-mail address: arne.holmgren@ki.se (A. Holmgren).
}

ways [6]. Today, a rapidly growing field is the role of Trx and TrxR in mammalian cell physiology and relation to specific functions. Recent reviews covering Trx [7] and mammalian TrxR [8] have been published. In the present article we will give an account of some aspects of $\operatorname{Trx}$ and TrxR with special reference to human diseases.

\section{Mammalian thioredoxin and thioredoxin reductase}

Purification of rat liver Trx and TrxR to homogeneity [9] demonstrated that mammalian TrxRs were larger and had a broader substrate specificity than the prokaryotic forms and that cytosolic Trxs contain three-structural SH-groups, which were sensitive to oxidation. Today, we know that TrxR is a selenoenzyme with three isoforms TrxR1 in the cytosol, TrxR2 in mitochondria and TrxR3 or TGR (thioredoxin glutathione reductase) present primarily in testis [8]. Trx1 is a cytosolic and extracellular enzyme whereas Trx2 exists in mitochondria [7]. Compared to prokaryotic Trx systems the most remarkable evolution is that TrxR is a large selenoenzyme, which is radically different from the smaller specific enzyme present in all bacteria, fungi, and plants. A lot of research has focused on the structure and mechanism of TrxR and particularly its reaction with drugs presently used in treating inflammation or cancer $[7,8]$.

\section{Thioredoxin system as a general protein disulfide reductase}

The most general description of the Trx system is its role as a protein disulfide reductase (Fig. 1). The enzyme operates by taking 


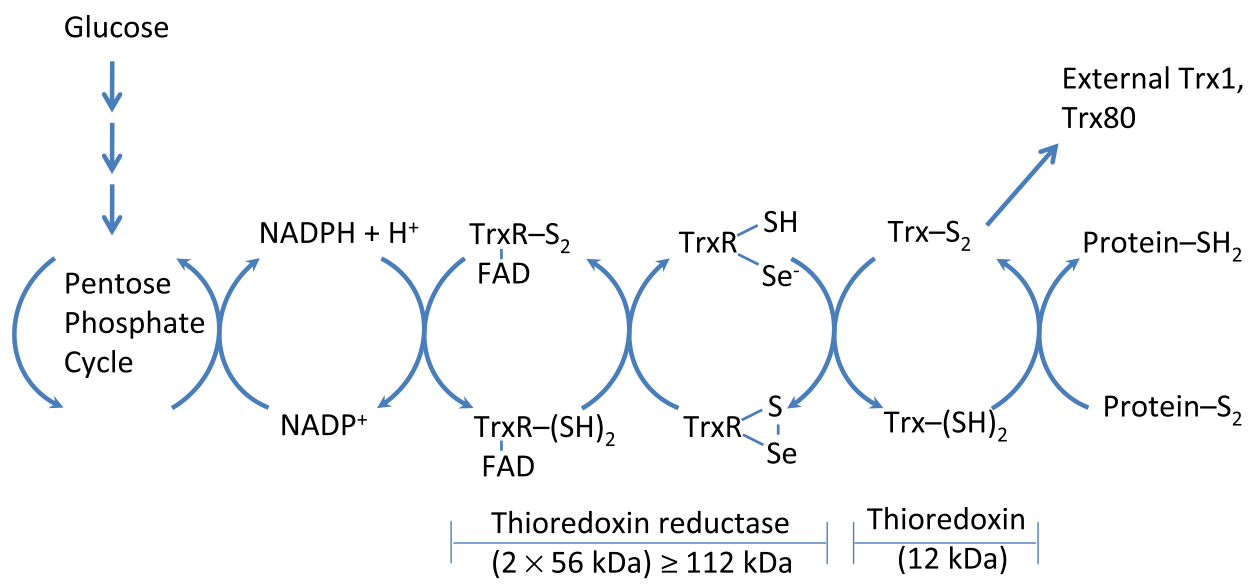

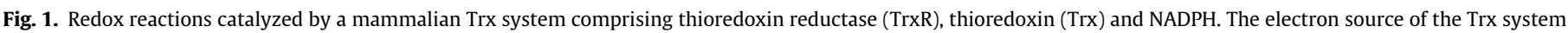

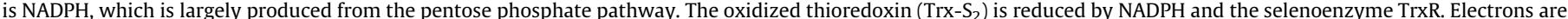

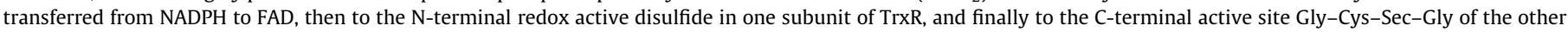

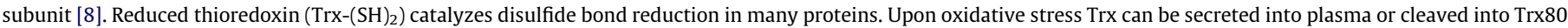
lacking the C-terminal 20 or 24 amino acid residues [10].

electrons from NADPH and via TrxR these are transferred to the active site of Trx, which is the general disulfide reductase. Mammalian TrxR consists of two subunits in a head to tail arrangement with a large number of splice forms [8] and the electrons from NADPH reduce a redox active disulfide and transfer them to the C-terminally located active site selenothiol located in the sequence Gly-Cys-Sec-Gly, which is conserved in all isoforms of TrxR. From there electrons move to Trx, which reduces protein disulfides or other substrates.

As seen in Fig. 1, Trx is secreted to plasma and is also present in a truncated form (Trx80), which has activity as a growth factor from monocytes inducing at Th1 response in the presence of PBMC cells via IL12 [10]. Plasma levels of Trx1 and also of TrxR1 have been used as a marker of inflammation, cancer and HIV infection $[11,7]$.

The structures of $\operatorname{Trx}-\mathrm{S}_{2}$ and $\operatorname{Trx}-(\mathrm{SH})_{2}$ are overall similar and a localized conformational change occurs on reduction of $\operatorname{Trx}-\mathrm{S}_{2}[12]$. The catalytic mechanism of Trx [12] involved docking to a target protein via a hydrophobic surface area and a nucleophilic attack by the active site Cys32 thiolate to form a transition state mixed disulfide [5]. Recently, the chemistry of Trx catalysis has been studied by single-molecule force-clamp spectroscopy [13]. The results, following application of mechanical force in the range of 25$600 \mathrm{pN}$, detected two alternative forms of the catalytic reaction, the first requiring a reorientation of the substrate disulfide bond and causing a shortening of the substrate polypeptide by about $0.8 \AA$ [13]. The second form of catalysis involved elongating the disulfide bond by about $0.2 \AA$ [13]. The results suggest that the Trx active site regulates the geometry of the substrate disulfide atoms with sub-Ångström precision for efficient catalysis [13]. The conformation of the substrate disulfide under conditions of oxidative stress or mechanical injury such as in cardiovascular disease, may thus impact on the efficiency of Trx system catalysis. The Michaelis-Menten type of reaction in Trx catalysis involves a binding surface area or groove [13]. Interestingly, eukaryotic Trxs from $\mathrm{X}$-ray and NMR structures have binding grooves that are several Ångströms deeper than those of bacterial origin and this is reflected in the mechanisms of disulfide reduction as revealed by single-molecule force-clamp spectroscopy [14]. A shallow binding groove as in human mitochondrial Trx2, a Trx of bacterial origin allows the substrate to be mobile [14]. In contrast the deeper groove found in human Trx1 in the cytosol or nucleus [14] tends to freeze the substrate in a much smaller range of conformations. This evo- lution of the chemistry of Trx catalysis by deepening the groove may have occurred to improve the specificity of substrate-enzyme interactions [14] at the same time as a much larger number of potential new functions and targets evolved.

\section{The large number of functions of Trx and TrxR related ultimately to disease}

Today, there are a large number of functions for Trx and TrxR in surprisingly many biological systems [6,7]. This is a reflection of the fact that Trx exists in all living cells and has a long evolutionary history in parallel with DNA as a genetic material, in the development of oxygen metabolism and defense against oxidative stress and the emergence of complicated physiological functions including the use of redox signaling with oxidants like hydrogen peroxide and nitric oxide. As illustrated in Fig. 2 there are numerous systems with thiol-dependent redox mechanisms, which are related to important pathological states and human diseases. Below, we will comment and discuss some recent results regarding functions of Trx and TrxR with particular emphasis on understanding molecular details, diagnostic opportunities, and drug mechanisms.

Ribonucleotide reductase (RNR) catalyzes the rate limiting step in deoxyribonucleotide synthesis. This is essential for DNA replication and repair. In the S-phase the mammalian cell RNR comprises a cytosolic complex of the two dimeric proteins: the R1-protein containing redox active cysteine residues, substrate binding sites as well as allosteric sites for regulation of overall activity and substrate specificity [15] and the R2-protein harbouring a tyrosyl free radical. Each enzyme turnover generates a disulfide in the active site of R1, which has to be reduced by Trx or glutaredoxin [16]. However, the immediate substrate for Trx is a C-terminally located shuttle disulfide/dithiol [17]. The results showed that Trx1 acts by a classical disulfide reductase mechanism [17] in contrast to the glutaredoxin system, which acts by a glutathionylation mechanism $[17,18]$. The $V_{\max }$ value for Trx was higher as well as the $k_{\mathrm{m}}$ than those for glutaredoxin, resulting in an overall catalytic efficiency $\left(K_{\text {cat }} / k_{\mathrm{m}}\right)$ that was similar [17]. Ongoing research tests the activity of p53R2 and R1, which is the enzyme present in postmitotic cells and required for dNTP synthesis for DNA repair and mitochondrial DNA synthesis and turnover [19]. Most cancer cells have a high level of expression of Trx and TrxR, which has been assumed to be a protection against apoptosis and promote cell growth [20-23]. TrxR is an important target for cancer therapy (Fig. 3). With some 


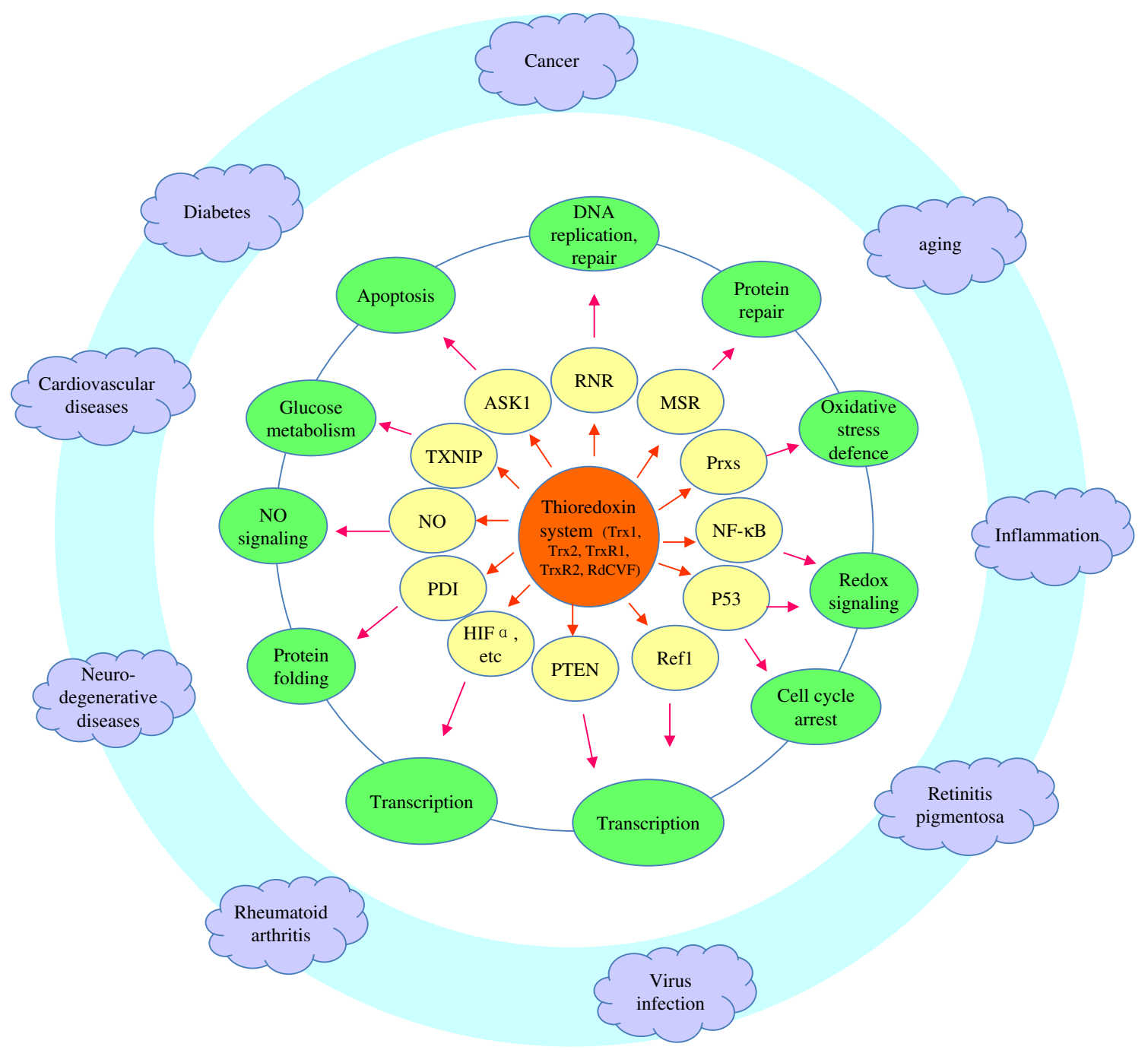

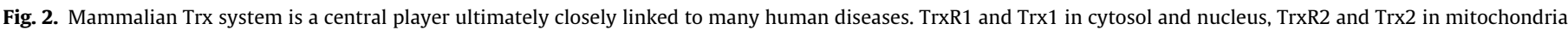

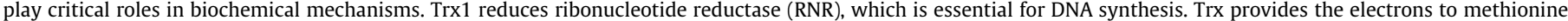

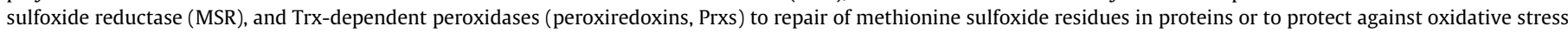

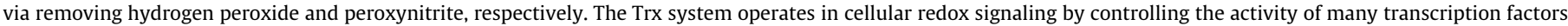

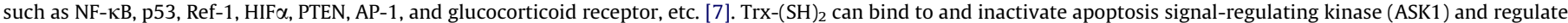

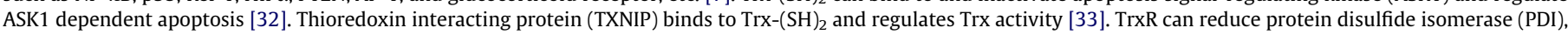

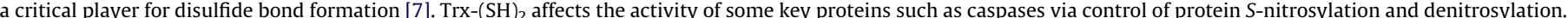

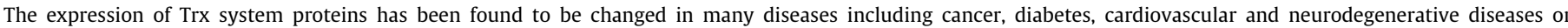

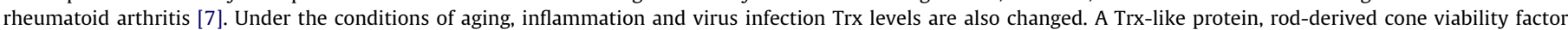
(RdCVFL) has been shown to be an essential factor to prevent cone loss, which induces retinitis pigmentosa [39].

drugs the enzyme is converted to an NADPH oxidase promoting cell death $[7,8]$. However, some malignant cells have low or undetectable level of Trx and in these cells probably glutaredoxin or potentially unknown electron donors are involved in ribonucleotide reduction and DNA synthesis [24,25]. One important objective in future research will be to determine the nature of the electron donor (Trx or glutaredoxin) in order to use the right inhibitors of either TrxR or the glutaredoxin system. We have recently established sensitive fluorescent assays for Trx, TrxR, and glutaredoxin using fluorescent substrates (S.J. Montano, J. Lu, A. Holmgren, manuscript in preparation) which should enable simple measurements of the level of activity of the Trx or glutaredoxin systems.

The demand for turnover of the Trx system (Figs. 1 and 2 ) is obviously very large for the RNR reaction, where it can be estimated that an S-phase T-cell generates more than 100,000 disulfides per second from ribonucleotide reductase to satisfy the requirement of deoxyribonucleotides for DNA replication [17]. This is by orders the fastest potential turnover of the Trx system. Other systems where substantial turnovers are required to protect cells from oxidative damage is for peroxiredoxins (Prx), or Trx peroxidases, which with six isoforms, occur both in the cytosol and the mitochondria [26]. In particular, the Prx3 of mitochondria working with TrxR2 and Trx2 removes peroxides from hydrogen peroxide generated via SOD and the superoxide in the electron transport chain [27]. Similarly, the methionine sulfoxide reductases operate by a mechanism generating a disulfide after each methionine sulfoxide residue is repaired back to methionine [7]. The importance of this system is shown by the shortened lifespan of mice with knockout of MsrA gene [28]. In line with this study transgenic mice overexpressing human Trx1 are reported to have a longer lifespan [29] and are protected against oxidative stress diseases [7].

A large number of transcription factors are regulated via redox signaling by Trx, TrxR, and Ref-1 [7,30]. As illustrated in Fig 2, NF- $\kappa$ B, p53, Ref-1, PTEN, AP-1 and a long list of other factors 


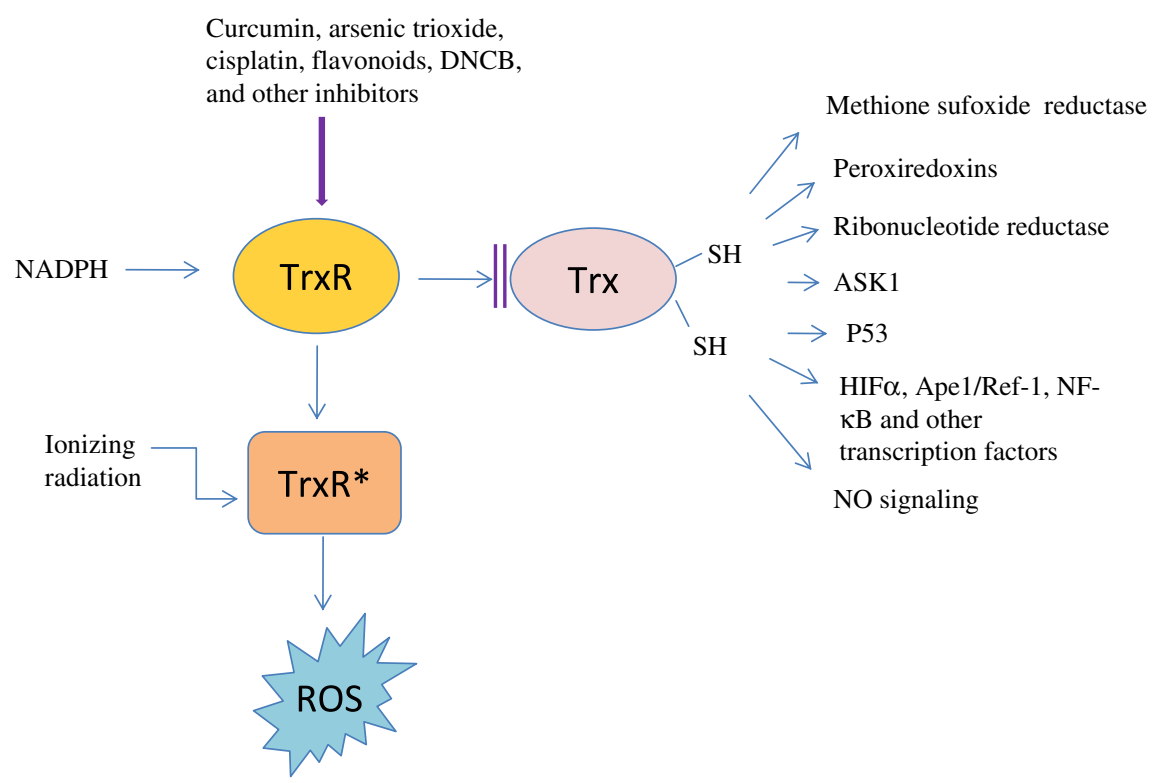

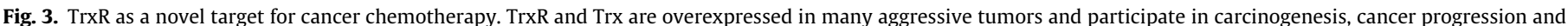

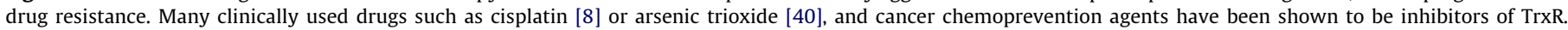

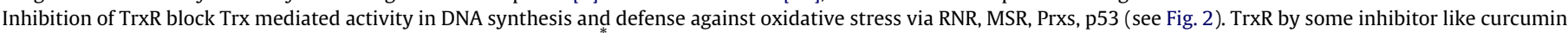

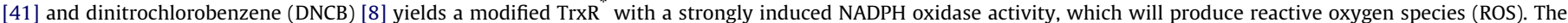
conversion of TrxR into pro-oxidant and a ROS source contributes the radiosensitization of cucurmin for some malignant tumors [42].

including estrogen receptors $\alpha$ and $\beta$ [31] belonging to this category. Trx operates mainly to keep the proteins active by reducing critical Cys residues either for activity of the transcription factor to bind to DNA or to control enzyme activity like for PTEN [7]. There are lots of details to be understood about the precise interaction between Ref-1 and Trx 1 and the movement of proteins from the cytosol to the nucleus in response to signals and in different cell types.

\section{Binding of reduced thioredoxin to other proteins}

The structure of reduced and oxidized Trx are similar as determined by structural biology methods like NMR and X-ray crystallography [12]. However, the NMR measurements showed that there are more structural isoforms and higher mobility around the active site in the reduced form of Trx. A critical example of this in mammalian cells is that only fully reduced Trx binds to apoptosis signal-regulating kinase 1 (ASK1) [32] and the same is true for thioredoxin interacting protein (TXNIP) also called TBP2 or VDUP-1 [33]. Via ASK1, reduced Trx1 will control cell death, since the downstream signaling of ASK1, a MAP kinase-kinase-kinase will lead to induction of apoptosis [34]. TXNIP is a tumor suppressor, which controls the activity of Trx system and is downregulated in tumor cells. It is also upregulated by glucose and has been implicated in e.g. $\beta$-cell death during diabetes [35].

Toxicity of mercury in cells involves binding of the metal to both reduced Trx1 and TrxR1 with loss of activity [36]. Signaling in mammalian cells by oxidants like hydrogen peroxide and nitric oxide results in protein modification via the formation of sulphenic acid residues or $S$-nitrosylated proteins [6,7]. Human $\operatorname{Trx} 1$ is itself regulated by formation of an inactive monomeric 2 -disulfide form via hydrogen peroxide oxidation, which is reversible by autocatalytic reduction [37]. The major protein denitrosylating activity in cells is by Trxs [38]. This opens up an universe of protein redox modifications in a living cell with the Trx system as one player and glutathione and the glutaredoxin systems as another [18] and the potential use of the redoxins as drugs to combat oxidative stress-related diseases.

\section{Acknowledgments}

Supported by Grants from the Swedish Research Council Medicine (3529), Swedish Cancer Society (961) and the K.A. Wallenberg Foundation.

\section{References}

[1] T.C. Laurent, E.C. Moore, P. Reichard, Enzymatic synthesis of deoxyribonucleotides. IV. Isolation and characterization of thioredoxin, the hydrogen donor from Escherichia coli B, J. Biol. Chem. 239 (1964) 3436-3444.

[2] E.C. Moore, P. Reichard, L. Thelander, Enzymatic synthesis of deoxyribonucleotides. V. Purification and properties of thioredoxin reductase from Escherichia coli B, J. Biol. Chem. 239 (1964) 3445-3452.

[3] A. Holmgren, Thioredoxin: 6. The amino acid sequence of the protein from Escherichia coli B, Eur. J. Biochem. 6 (1968) 475-484.

[4] A. Holmgren, B.-O. Söderberg, H. Eklund, C.-I. Brändén, Three-dimensional structure of Escherichia coli thioredoxin- $\mathrm{S}_{2}$ to 2.8 A resolution, Proc. Natl. Acad. Sci. USA 72 (1975) 2305-2309.

[5] A. Holmgren, Thioredoxin, Annu. Rev. Biochem. 54 (1985) 237-271.

[6] Y. Meyer, B.B. Buchanan, F. Vignols, J.-P. Reichheld, Thioredoxins and glutaredoxins: unifying elements in redox biology, Annu. Rev. Genet. 43 (2009) 335-367.

[7] C.H. Lillig, A. Holmgren, Thioredoxin and related molecules: from biology to health and disease, Antioxid. Redox Signal. 9 (2007) 25-47.

[8] E.S. Arner, Focus on mammalian thioredoxin reductases-important selenoproteins with versatile functions, Biochim. Biophys. Acta 6 (2009) 495-526.

[9] M. Luthman, A. Holmgren, Rat liver thioredoxin and thioredoxin reductase: purification and characterization, Biochemistry 21 (1982) 6628-6633.

[10] K. Pekkari, J. Avila-Carino, A.. Bengtsson, R. Gurunath, A. Scheynius, A. Holmgren, Truncated thioredoxin (Trx80) induces interleukin 12 production and enhances CD14 expression in human monocytes, Blood 97 (2001) 31843190.

[11] H. Nakamura, S. DeRosa, M. Roederer, J. Yodoi, A. Holmgren, L.A. Herzenberg, L.A. Herzenberg, Chronic elevation of plasma thioredoxin: inhibition of chemotaxis and curtailment of life expectancy in AIDS, Proc. Natl. Acad. Sci. USA 98 (2001) 2688-2693.

[12] A. Holmgren, Thioredoxin structure and mechanism: conformational changes on oxidation of the active site sulfhydryls to a disulfide, Structure 3 (1995) 239-243.

[13] A.P. Wiita, R. Perez-Jimenez, K.A. Walther, F. Grater, B.J. Berne, A. Holmgren, J.M. Sanchez-Ruis, J.M. Fernandez, Probing the chemistry of thioredoxin catalysis with force, Nature 450 (2007) 124-127.

[14] R. Perez-Jimenez, J. Li, P. Kosuri, I. Sanchez-Romero, A.P. Wiita, D. RodriguezLarrea, A. Holmgren, A. Miranda-Vizuete, K. Becker, S.-H. Cho, J. Beckwith, E. Gelhaye, J.-P. Jacquot, E. Gaucher, J.M. Sanchez-Ruiz, B.J. Berne, J.M. Fernandez, 
Diversity of chemical mechanism in thioredoxin catalysis revealed by singlemolecule force spectroscopy, Nat. Struct. Mol. Biol. 16 (2009) 890-896.

[15] P. Nordlund, P. Reichard, Annu. Rev. Biochem. 75 (2006) 681-706.

[16] A. Holmgren, Thioredoxin and glutaredoxin systems, J. Biol. Chem. 264 (1989) 13963-13966.

[17] F. Zahedi Avval, A. Holmgren, Molecular mechanisms of thioredoxin and glutaredoxin as hydrogen donors for mammalian S-phase ribonucleotide reductase, J. Biol. Chem. 284 (2009) 8233-8240.

[18] C.H. Lillig, C. Berndt, A. Holmgren, Glutaredoxin systems, Biochem. Biophys. Acta 1780 (2008) 1304-1317.

[19] G. Pontarin, A. Fijolek, P. Pizzo, P. Ferraro, C. Rampazzo, T. Pozzan, L. Thelander, P.A. Reichard, V. Bianchi, Ribonucleotide reduction is a cytosolic process in mammalian cells independently of DNA damage, Proc. Natl. Acad. Sci. USA 46 (2008) 17801-17806.

[20] A. Baker, C.M. Payne, M.M. Briehl, G. Powis, Thioredoxin, a gene found overexpressed in human cancer, inhibits apoptosis in vitro and in vivo, Cancer Res. 22 (1997) 5162-5167.

[21] G. Powis, D. Mustacich, A. Coon, The role of the redox protein thioredoxin in cell growth and cancer, Free Radic. Biol. Med. 3-4 (2000) 312-322.

[22] H. Nakamura, H. Masutani, J. Yodoi, Extracellular thioredoxin and thioredoxinbinding protein 2 in control of cancer, Semin. Cancer Biol. 6 (2006) 444-451.

[23] S. Urig, K. Becker, On the potential of thioredoxin reductase inhibitors for cancer therapy, Semin. Cancer Biol. 6 (2006) 452-465.

[24] K. Kahlos, Y. Soini, M. Säily, P. Koistinen, S. Kakko, P. Pääkö, A. Holmgren, V.L. Kinnula, Upregulation of thioredoxin and thioredoxin reductase in human malignant pleural mesothelioma, Int. J. Cancer 95 (2001) 198-204.

[25] Y. Soini, K. Kahlos, U. Näpänkangas, R. Kaarteenaho-Wiik, M. Säily, P. Koistinen, P. Päkkö, A. Holmgren, V.L. Kinnula, Widespread expression of thioredoxin and thioredoxin reductase in non-small cell lung carcinoma, Clin. Cancer Res. 7 (2001) 1750-1757.

[26] S.G. Rhee, H.Z. Chae, K. Kim, Peroxiredoxins: a historical overview and speculative preview of novel mechanisms and emerging concepts in cell signaling, Free Radic. Biol. Med. 12 (2005) 1543-1552.

[27] S. Matsushima, T. Ide, M. Yamato, H. Matsusaka, F. Hattori, M. Ikeuchi, T. Kubota, K. Sunagawa, Y. Hasegawa, T. Kurihara, S. Oikawa, S. Kinugawa, H. Tsutsui, Overexpression of mitochondrial peroxiredoxin-3 prevents left ventricular remodeling and failure after myocardial infarction in mice, Circulation 14 (2006) 1779-1786.

[28] J. Moskovitz, S. Bar-Noy, W.M. Williams, J. Requena, B.S. Berlett, E.R. Stadtman, Methionine sulfoxide reductase (MsrA) is a regulator of antioxidant defense and lifespan in mammals, Proc. Natl. Acad. Sci. USA 23 (2001) 12920-12925.

[29] A. Mitsui, J. Hamuro, H. Nakamura, N. Kond, Y. Hirabayashi, S. IshizakiKoizumi, T. Hirakawa, T. Inoue, J. Yodoi, Overexpression of human thioredoxin in transgenic mice controls oxidative stress and life span, Antioxid. Redox Signal. 4 (2002) 693-696.
[30] S. Gromer, S. Urig, K. Becker, The thioredoxin system - from science to clinic Med. Res. Rev. 1 (2004) 40-89.

[31] A.E. Damdimopoulos, A. Miranda-Vizuete, E. Treuter, J.-Å. Gustafsson, G. Spyrou, An alternative splicing variant of the selenoprotein thioredoxin reductase is a modulator of estrogen signaling, J. Biol. Chem. 279 (2004) 38721-38729.

[32] M. Saitoh, H. Nishitoh, M. Fujii, K. Takeda, K. Tobiume, Y. Sawada, M. Kawabata, K. Miyazono, H. Ichijo, Mammalian thioredoxin is a direct inhibitor of apoptosis signal-regulating kinase (ASK)1, EMBO J. 9 (1998) 2596-2606.

[33] J. Yoshioka, P.C. Schulze, M. Cupesi, J.D. Sylvan, C. MacGillvray, J. Cannon, H. Huang, R.T. Lee, Thioredoxin-interacting protein controls cardiac hypertrophy through regulation of thioredoxin activity, Circulation 21 (2004) 2581-2586.

[34] M. Niso-Santano, R.A. González-Polo, J.M. Bravo-San Pedro, R. Gómez-Sánchez, I. Lastres-Becker, M.A. Oritz-Oritz, G. Soler, J.M. Morán, A. Cuadrado, J.M. Fuentes, Activation of apoptosis signal-regulating kinase 1 is a key factor in paraquat-induced cell death. Modulation by the Nrf2/Trx axis, Free Radic. Biol. Med. (2010)

[35] J. Chen, G. Saxena, I.N. Mungrue, A.J. Lusis, A. Shalev, Thioredoxin-interacting protein: a critical link between glucose toxicity and beta-cell apoptosis, Diabetes 4 (2008) 938-944.

[36] C.M.L. Carvalho, E.-H. Chew, S.I. Hashemy, J. Lu, A. Holmgren, Inhibition of human thioredoxin system: a molecular mechanism of mercury toxicity, J. Biol. Chem. 283 (2008) 11913-11923.

[37] S.I. Hashemy, A. Holmgren, Regulation of the catalytic activity and structure of human thioredoxin 1 via oxidation and S-nitrosylation of cysteine residues, J Biol. Chem. 283 (2008) 21890-21898.

[38] M. Benhar, M.T. Forrester, D.T. Hess, J.S. Stamler, Regulated protein denitrosylation by cytosolic and mitochondrial thioredoxins, Science 5879 (2008) 1050-1054.

[39] R. Fridlich, F. Delalande, C. Jaillard, J. Lu, L. Poidevin, T. Cronin, L. Perrocheau, G. Millet-Puel, M.L. Niepon, O. Poch, A. Holmgren, A. Van Dorsselaer, J.A. Sahel, T. Léveillard, The thioredoxin-like protein rod-derived cone viability factor (RdCVFL) interacts with TAU and inhibits its phosphorylation in the retina, Mol. Cell Proteomics 6 (2009) 1206-1218.

[40] J. Lu, E.-H. Chew, A. Holmgren, Targeting thioredoxin reductase is a basis for cancer therapy by arsenic trioxide, Proc. Natl. Acad Sci. USA 104 (2007) 1228812293.

[41] J. Fang, J. Lu, A. Holmgren, Thioredoxin reductase is irreversibly modified by curcumin: a novel molecular mechanism for its anticancer activity, J. Biol Chem. 280 (2005) 25284-25290.

[42] P. Javvadi, L. Hertan, R. Kosoff, T. Datta, J. Kolev, R. Mick, S.W. Tuttle, C. Koumenis, Thioredoxin reductase-1 mediates curcumin-induced radiosensitization of squamous carcinoma cells, Cancer Res. 5 (2010) 1941 1950. 